\title{
Interleukin-6 signaling regulates hematopoietic stem cell emergence
}

\author{
Ruxiu Tie ${ }^{1,2,3,4,5}$, Honghu Li ${ }^{1,2}$, Shuyang Cai ${ }^{1,2}$, Zuyu Liang ${ }^{1,2}$, Wei Shan ${ }^{1,2}$, Binsheng Wang ${ }^{1,2}$, Yamin Tan ${ }^{1,2}$, \\ Weiyan Zheng ${ }^{1,2}$ and He Huang 1,2,3,4
}

\begin{abstract}
Hematopoietic stem cells (HSCs) produce all lineages of mature blood cells for the lifetime of an organism. In vertebrates, HSCs derive from the transition of the hemogenic endothelium (HE) in the floor of the embryonic dorsal aorta. Most recently, a series of proinflammatory factors, such as tumor necrosis factor-a, interferon- $\gamma$, and Toll-like receptor 4, have been confirmed to play a key role in HSC specification. However, the full complement of necessary signaling inputs remains unknown to date. Here, we show that interleukin-6R (IL6R) via IL6 is required and sufficient for HSC generation. We found that Notch activates IL6R by regulating its expression in the HE and in HSCs. The secretion of IL6 mainly originates from HSC-independent myeloid cells, but not from HSCs and their adjacent vascular endothelial cells. In addition, blocking IL6 signaling does not affect vascular development or the production of primitive erythrocytes. Taken together, our results uncover a previously obscure relationship between IL6 signaling and HSC production and provide new insights into HSC regeneration using proinflammatory factors in vitro.
\end{abstract}

\section{Introduction}

Adult hematopoietic stem cells (HSCs) have the ability to self-renew and differentiate into all blood and immune cells throughout life. In vertebrate embryos, naive HSCs arise de novo from the hemogenic endothelium (HE) in the ventral wall of the dorsal aorta ${ }^{1-4}$. Thus far, many signaling pathways have been implicated in this complex process. However, a more comprehensive understanding remains needed, partly because of the failure to reproduce healthy HSCs for HSC transplantation (HSCT) in vitro ${ }^{5-8}$.

Under stress-induced hematopoiesis, adult short-term HSCs and multipotent progenitor cells (MPPs) can directly sense bacterial and viral components and systematically elevate cytokine expression through Toll-like receptors $(\mathrm{TLRs})^{9-11}$. Numerous cytokines, such as

Correspondence: He Huang (huanghe@zju.edu.cn)

'Bone Marrow Transplantation Center, The First Affiliated Hospital, Zhejiang University School of Medicine, No. 79 Qingchun Road, 310003 Hangzhou,

China

${ }^{2}$ Institute of Hematology, Zhejiang University, No. 866 Yuhangtang Road, Hangzhou 310058, China

Full list of author information is available at the end of the article.

These authors contributed equally: Ruxiu Tie, Honghu Li, Shuyang Cai interleukin-6 (IL6), tumor necrosis factor- $\alpha$ (TNF- $\alpha$ ), interferon- $\gamma$ (IFN- $\gamma)$, granulocyte-macrophage colonystimulating factor (GM-CSF), and IFN- $\alpha$, have been identified to regulate the proliferation and differentiation of adult hematopoietic stem and progenitor cells (HSPCs) in a paracrine manner ${ }^{11}$. These studies prompted us to investigate whether these cytokines play a role in HSC production under physiological conditions. Most recently, several studies have demonstrated that proinflammatory signaling factors, including granulocyte-CSF(G-CSF), TNF- $\alpha$, IFN- $\gamma$, and TLR4, can positively specify embryonic HSC fate ${ }^{12-16}$. Importantly, all of these proinflammatory factors are functionally associated with Notch signaling.

IL6 is a prominent proinflammatory cytokine that plays a pivotal role in controlling immunity and inflammation ${ }^{17,18}$. IL6 exerts its functions via the engagement of the type 1 cytokine a-receptor subunit (IL6R) and the signal-transduction $\beta$-receptor subunit (GP130) ${ }^{19-22}$. IL6, IL6R, and GP130 (Il6, Il6r, and Gp130 utilizing zebrafish nomenclature) are well conserved in all vertebrate organisms. The targeted disruption of GP130 leads to embryonic lethality, presenting hypoplastic ventricular 
myocardium and reduced HSPCs in the liver, whereas more detailed information is absent ${ }^{23}$. It is well known that GP130 is a common signal transducer for IL6 family of cytokines, including IL6, IL11, IL27, leukemia inhibitory factor, oncostatin $M$, ciliary neurotrophic factor, cardiotrophin 1, and cardiotrophin-like cytokine. Thus, we fail to discern which signaling pathway may participate in embryonic hematopoiesis. One report demonstrates that adult HSPCs from Il6-deficient mice have defective capacities in proliferation and self-renewal compared with those of wild-type controls ${ }^{24}$. However, it is still unknown whether IL6 signaling affects embryonic HSC emergence. With the diverse combinations with other classical factors, IL6 has been used to promote the proliferation of HSPCs in vitro ${ }^{25-27}$. Most recently, another study demonstrated that platelet-derived growth factor receptor- $\beta$ signaling promotes embryonic HSC production by regulating il6 expression ${ }^{28}$, but this study did not depict the role of Il6 signaling in HSC emergence. All of the evidence above suggests that Il6 signaling may participate in embryonic HSC specification and maintenance, and a comprehensive blueprint needs to be explored.

During embryonic development, hematopoiesis occurs in at least two waves, known as primitive and definitive hematopoiesis, which are conserved in all vertebrates. Primitive hematopoiesis produces mainly primitive erythrocytes, macrophages (MFs) and megakaryocytes $(\mathrm{Mks})^{29}$. Definitive erythromyeloid progenitors (EMPs) ${ }^{30}$ emerge distinct from both primitive hematopoiesis and HSCs, which produces mainly definitive erythrocytes, MFs, Mks, and neutrophils ${ }^{29,31}$. Zebrafish have emerged as a model for the study of hematopoietic disorders and embryonic hematopoiesis ${ }^{32-35}$. Here, we use the model to present a previously unappreciated requirement for Il6 signaling in HSC specification and emergence. We found that Notch activates $I l 6 r$ by regulating its expression in the HE and in HSCs, and Il6 is mainly secreted by neutrophils and MFs from primitive and definitive EMP hematopoiesis. Altogether, our study reveals an undiscovered function of Il6 signaling in the birth of HSCs under nonpathogenic conditions.

\section{Materials and methods}

\section{Zebrafish husbandry and strains}

Zebrafish strains were raised as described ${ }^{36}$, and the adult fish were maintained in accordance with the relevant guidelines of the Laboratory Animal Center of Zhejiang University. The study was approved by the Institutional Animal Care and Use Committee of the Laboratory Animal Center, Zhejiang University (Hangzhou, China). See the Supplemental Information for a description of the transgenic lines used in this study (Table S1).

\section{Morpholino and mRNA injections}

Antisense morpholinos (MOs) used in our study were purchased from Gene Tools, including Standard Control MO (Gene Tools), irf8 $\mathrm{MO}^{37}$, pu.1 $\mathrm{MO}^{38}$, tnfo $\mathrm{MO}^{13}$, notch1a $\mathrm{MO}^{13}$, il6 MO, and il6r MO (this work). The MOs were diluted in diethyl pyrocarbonate-treated water at a concentration of $0.2 \mathrm{mM}$ (Standard-MO), $0.5 \mathrm{mM}$ (tnfo MO), $1.2 \mathrm{mM}$ (notch1a MO), $0.6 \mathrm{mM}$ (il6 MO), $0.4 \mathrm{mM}$ (il6r MO), $1.4 \mathrm{mM}$ (irf8 MO), and $2 \mathrm{mM}$ (pu.1 $\mathrm{MO}$ ) with phenol red solution, and $1 \mathrm{nl}$ was injected into the yolk ball of one- or two-cell-stage embryos. The MO sequences are listed in Table S2. The iI6r MO validation was performed using reverse transcription-polymerase chain reaction (Table S3).

For messenger RNA (mRNA) generation, the total RNA of embryos at $28 \mathrm{hr}$ postfertilization (hpf) was isolated from MO-injected embryos and reverse transcribed into complementary DNA (cDNA). Two primers (Table S4) were used to amplify the $i l 6 r$ opening reading frame. The PCR product was cloned into the $\mathrm{pCS} 2+$ vector and confirmed by bidirectional sequencing (Vazyme). The pCS2 ${ }^{+}-i l 6 r$ vector was linearized by NotI and purified (Takara Purification Kit). Capped il6r mRNA was produced using the SP6 mMESSAGE mMACHINE Kit (Life Technologies), and then $200 \mathrm{pg}$ purified il6r mRNA was injected into one-cell stage embryos at the yolk/cytoplasm boundary for rescue experiments.

\section{Whole-mount RNA in situ hybridization}

Probes for the runx1, cmyb, kdrl, efnb2a, dlc, gata1a, mpx, l-plastin, rag1, and foxn1 transcripts were generated using a DIG RNA Labeling Kit (Roche Applied Science) from linearized plasmids. Whole-mount in situ hybridization (WISH) was carried out as described previously ${ }^{39}$. The embryos were observed using a Leica M165C stereomicroscope and imaged with a DFC295 color digital camera (Leica). Qualitative phenotypes for individual embryos $(n \geq 10$ embryos/condition, $n \geq 3$ replicate clutches unless otherwise indicated) were assessed as relatively high (up)/medium (normal)/low (down)/absent in expression compared with control siblings at noted stages and were depicted graphically as the percentage falling into each of three phenotypic expression bins; "medium" expression was the most representative qualitative phenotype in the normal bell curve distribution of each control group per experiment.

\section{Enumeration of HSCs}

Confocal microscopy was performed on cmyb:GFP; $k d r l$ : $m$ Cherry double-transgenic animals ${ }^{1}$, tp1:eGFP; $k d r l$ : $m$ Cherry double-transgenic animals, and mpeg1:eGFP transgenic animals. $\mathrm{Z}$ sections of the DA region or whole embryos were captured on a Leica SP5 microscope (Leica) 
using Volocity Acquisition, Visualization, and Restoration software (Improvision) and were manually counted.

Fluorescent visualization of HSPCs, neutrophils, and T cells

To visualize HSPCs, neutrophils, and T cells, we used these transgenic models, including cd41:eGFP; lyz:dsred embryos at $72 \mathrm{hpf}$ and $l c k: G F P$ larvae at 5 days postfertilization (dpf), respectively, were anesthetized in tricaine $(200 \mu \mathrm{g} / \mathrm{ml})$ and observed using a Leica MZ16FA stereomicroscope.

\section{FACS and quantitative real-time PCR}

Fluorescence-activated cell sorting (FACS) analysis was performed using fli1a:eGFP, mpx:eGFP, and mpeg1: eGFP transgenic embryos. First, 50-100 embryos were stored on ice in $500 \mu \mathrm{l}$ phosphate-buffered saline (PBS) containing $2 \%$ fetal bovine serum and dissociated using a P1000 pipette. The resulting suspension was filtered with a $40 \mu \mathrm{m}$ cell strainer and resuspended in $1 \times$ PBS. Then, we isolated target cells by using a FACSAria cell sorter (BD Biosciences, San Jose, CA). Subsequently, we used an RNeasy Mini Kit (Qiagen) to extract mRNA. During the process, $500 \mathrm{ng}$ polyinosinic acid potassium salt (Sigma) was added to the RLT buffer for each sample. cDNA was synthesized using a Quantitect cDNA Synthesis Kit (Qiagen) and diluted five times as the template. Quantitative PCR (qPCR) was performed using the Bio-Rad CFX96 real-time PCR system. Relative expression was calculated by the $2^{-\triangle \triangle \mathrm{C}(\mathrm{T})}$ method, and ef1a was used as the housekeeping gene ${ }^{13}$. The PCR primers used in this study are listed in supplemental Table S5.

\section{Detection of apoptotic cell death by TUNEL labeling}

TUNEL assays were performed as described previously ${ }^{40}$. The fli1a:eGFP transgenic embryos were fixed in $4 \%$ paraformaldehyde (PFA) at $4{ }^{\circ} \mathrm{C}$ overnight and then dehydrated in $100 \%$ methanol at $-20^{\circ} \mathrm{C}$ for at least $2 \mathrm{~h}$. After gradual rehydration, the embryos were washed three times with PBST and treated with proteinase $\mathrm{K}(10 \mu \mathrm{g} / \mathrm{ml})$ for $10 \mathrm{~min}$. At room temperature, the permeabilized embryos were refixed in $4 \%$ PFA for $20 \mathrm{~min}$. After washing 3-6 times with PBST, the embryos were incubated with a mixture containing $45 \mu \mathrm{l}$ labeling solution and $5 \mu$ l enzyme solution (In Situ Cell Death Detection Kit TMR Red; Roche) at $4{ }^{\circ} \mathrm{C}$ overnight. Finally, after washing three times with PBST, the stained embryos were imaged by confocal microscopy.

\section{Chemical treatment}

Experimental embryos were exposed to the Notchspecific inhibitor DAPT ( $N$-[ $N$-(3,5-difluorophenacetyl)lalanyl]-S-phenylglycine $t$-butyl ester) at $100 \mu \mathrm{M}$ (dissolved in dimethyl sulfoxide (DMSO), Sigma) from the 10-somite stage to 48 or $72 \mathrm{hpf}$ in multiwell plates of Danieau's solution. Siblings treated with 1\% DMSO in Danieau's solution were regarded as controls.

\section{Statistical analyses}

Statistical analysis was performed using the GraphPad Prism software (GraphPad). Two-tailed Student's $t$ tests were used for pairwise comparisons. In all statistical figures, solid red bars represent the mean, and error bars denote the SEM. The data are presented as the means \pm SEM. ${ }^{*} P<0.05$ was considered statistically significant, ${ }^{* *} p<0.01,{ }^{* * * *} p<0.001,{ }^{* * * * * *} p<0.0001$; n.s., not significant.

\section{Results}

II6 signaling through il6r is required for HSC production

Most recently, several studies in mouse and zebrafish embryos demonstrated that proinflammatory factors are required for HSC emergence ${ }^{12-14,16}$. IL6 is implicated in adult HSC proliferation and differentiation, but its detailed function in embryonic HSC development remains unclear. One recent published report isolated zebrafish $k d r l^{+} \mathrm{cmyb}^{-}$vascular endothelial cells (ECs) and $\mathrm{kdrl}^{+} \mathrm{cmyb}^{+}$HSCs from double-transgenic kdrl:mCherry and cmyb:eGFP embryos at $36 \mathrm{hpf}$ by FACS and performed qPCR for il6, il6r, and gp130. The transcript il6r was enriched in HSCs compared to ECs, while the expression differences in the other two transcripts were not observed between the two groups ${ }^{28}$. To further investigate the expression of these genes, we analyzed published single-cell transcriptomic data on mouse embryonic HSCs at different stages ${ }^{41}$. Compared to vascular ECs, the expression of il6r and gp130 was significantly higher in developing HSCs, whereas il6 was too low to be detected in HSCs at all time points (Fig. S1). These results suggest that Il6 may play an important role in embryonic HSC emergence in a paracrine way.

To investigate whether Il6 signaling was required for HSC specification, we performed loss-of-function experiments for Il6 and its receptor Il6r using targeted MOs. In the zebrafish embryo, HSCs are marked by the expression of $c m y b$ along axial vessels ${ }^{42}$. Using WISH, we found that the expression of cmyb in or near the floor of the DA region at $36 \mathrm{hpf}$ was dramatically reduced in Il6and Il6r-deficient embryos compared with their wild-type siblings (Fig. 1a, b), indicating that the action of Il6 through Il6r is required in HSC development. A similar result was observed by the quantitation of $c d 41: e G F P^{+}$ HSPCs in the caudal hematopoietic tissue (CHT) region at $72 \mathrm{hpf}^{43}$, as determined by fluorescence microscopy (Fig. 1c, d). The specificity of il6r MO was confirmed by splice product detection (Fig. S1B).

To further verify the reduction of HSCs in Il6- and Il6r-deficient embryos, we directly observed emerging HSCs from the floor of the DA region in kdrl:mCherry; 


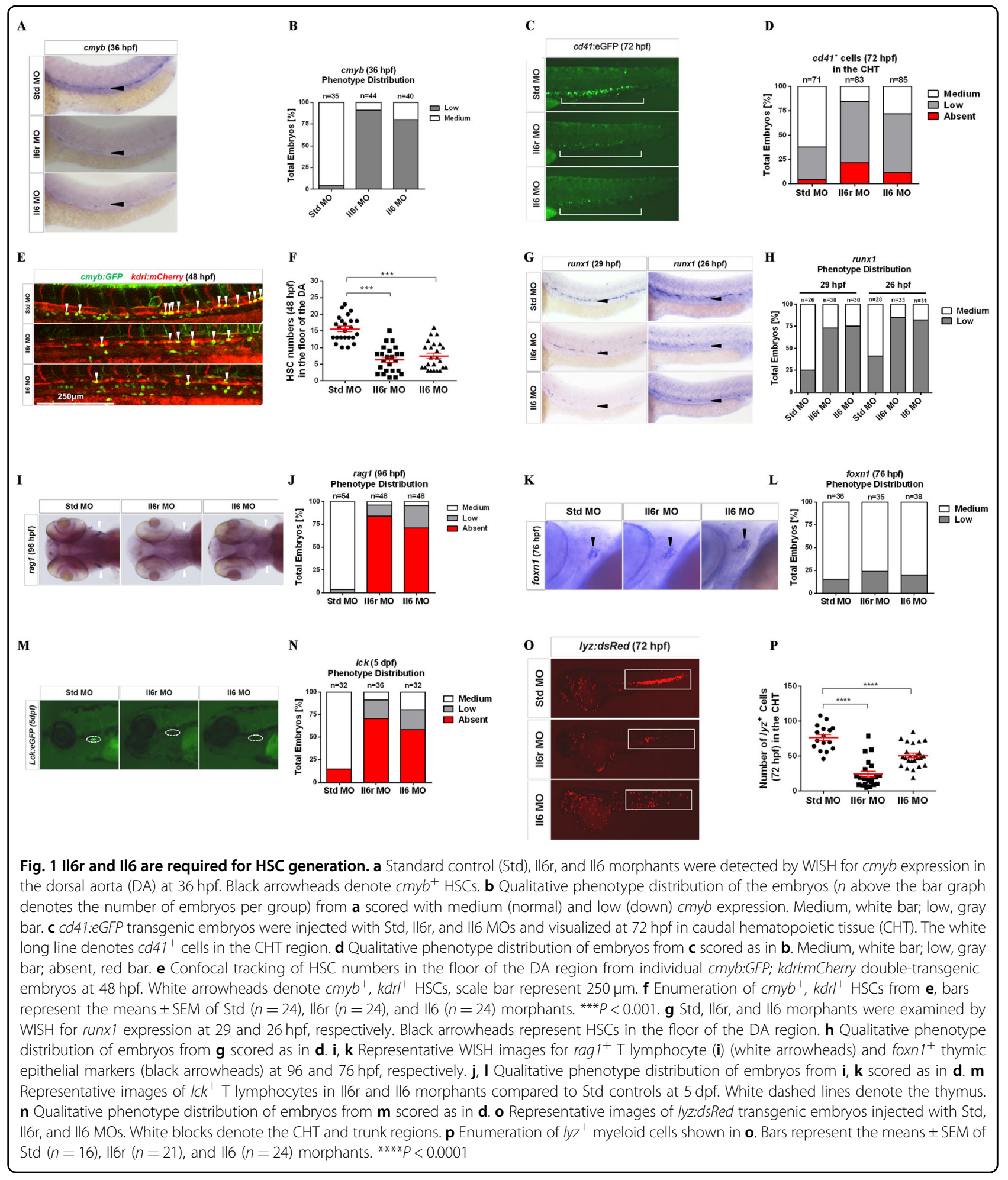

cmyb:GFP double-transgenic embryos at $48 \mathrm{hpf}$ by confocal microscopy ${ }^{1}$. Consistent with the above results, the number of $k d r l$; cmyb double-positive HSCs was significantly less than those in control embryos (Fig. 1e, f). This reduction might be due to a defect in the initial HSC specification. To address this question, we performed WISH to detect the expression of runxl, which is a nascent HSC marker at an earlier developmental stage ${ }^{1,2,4,44}$. The expression of runx 1 was significantly reduced in the aorta floor at 26 and $29 \mathrm{hpf}$ (Fig. 1g, h), indicating that 
Il6 signaling is required during the earliest stages of HSC specification.

We next investigated subsequent larval stages by detecting the expression of rag1 and $l c k^{45}$, two markers located in developing thymocytes because the $\mathrm{T}$ cell lineage derives exclusively from $\mathrm{HSCs}^{43,46}$. Rag1 expression was completely or nearly absent in Il6- and Il6r-deficient animals at $96 \mathrm{hpf}$ (Fig. 1i, j); however, the expression of the thymic epithelial cell marker foxn1 was normal in all morphants (Fig. 1k, l). The results were further confirmed by tracking $\mathrm{T}$ cell lineage development in lck:eGFP transgenic embryos at $5 \mathrm{dpf}$ (Fig. $1 \mathrm{~m}, \mathrm{n}$ ). ${ }^{45}$ In addition, we assessed myeloid lineage cell development using lyz:dsRed transgenic embryos in CHT, and the number of neutrophils was significantly reduced in Il6- and Il6r-deficient animals at $72 \mathrm{hpf}$ (Fig. 1o, p). Together, these results demonstrate that Il6 signaling via Il6r is essential for both the specification and subsequent maintenance of HSCs in the embryonic development stage.

II6 signaling through II6r is required for HSC-independent neutrophils but not MFs and primitive erythrocytes

To further dissect the role of Il6 signaling in embryonic hematopoiesis, we evaluated whether Il6 and its receptor Il6r were required for the first wave of hematopoiesis, also named the "primitive wave" due to the lack of upstream multipotent progenitors. The expression of l-plastin, a specific marker of myeloid cell lineage, was not significantly affected in the yolk sac of Il6 and Il6r morphants at $28 \mathrm{hpf}$ (Fig. 2a, b). Similar results were observed in terms of primitive neutrophils and MFs using the specific markers $m p x$ and mpeg1, respectively (Fig. 2a, c, d). Moreover, primitive erythropoiesis was unchanged in all morphants, as assayed by the expression of the erythrocyte-specific marker gatala at $24 \mathrm{hpf}$ (Fig. 2e, f).

In zebrafish embryos, secondary wave hematopoiesis occurs in the posterior blood island (PBI) produced autonomously at $\sim 30 \mathrm{hpf}$, namely, definitive EMPs, which are distinct from both primitive hematopoiesis and definitive $\mathrm{HSCs}^{47}$. We found that the expression of $l$-plastin and $m p x$ was decreased significantly in the PBI and trunk regions of Il6- and Il6r-deficient embryos, but the same trend was not observed in mpeg1-positive transgenic embryos (Fig. 2a-d), suggesting that the development of HSC-independent neutrophils from EMPs is also dependent on Il6 signaling. Overall, these results indicate that Il6 signaling is dispensable for primitive hematopoiesis and indispensable for definitive EMPs in the zebrafish embryo.

\section{II6r- and II6-deficient embryos display normal vasculogenesis}

HSCs originate in aorta vessels, and many genes affecting vascular or arterial specification also control HSC development ${ }^{40,47}$. No remarkable vascular abnormalities were found in Il6 and Il6r morphants at $28 \mathrm{hpf}$ when assayed by WISH for the vascular endothelial specific marker $k d r l$ in this study (Fig. 3a, b). To further determine whether the reduction in HSCs resulted from impaired arterial specification, we used WISH to detect the expression of the arterial-specific markers efn $b 2 a$ and $d l c$ at $28 \mathrm{hpf}^{48}$. No obvious alterations were observed among the different groups (Fig. 3a, b). Taken together, these results indicate that Il6 signaling promotes HSC emergence under normal vasculature conditions.

To determine whether the loss of HSCs in the absence of Il6 signaling could be attributed to the apoptosis of ECs, we performed a TUNEL assay using fli1a:GFP embryos injected with il6r and il6 MOs. Using confocal microscopy, we found that Il6 and Il6r morphants at $30 \mathrm{hpf}$ did not have increased apoptotic ECs within the DA region (Fig. 3c) ${ }^{49}$. Additionally, we performed WISH for runx 1 in this experiment to confirm the reduction in HSCs in these embryos (Fig. 3d, e). Taken together, these results indicate that the defects of HSC specification in Il6- and Il6r-deficient embryos are not caused by apoptosis of ECs within the vasculature.

\section{II6r acts downstream of Notch signaling for HSC production}

Notch signaling plays a crucial role in HSC specification $^{50-52}$. Most recently, several proinflammatory factors have been implicated in HSC emergence, such as TNF- $\alpha$, INF- $\gamma$, and TLR4, all of which are tightly associated with the Notch pathway ${ }^{12-14}$. In addition, one report has demonstrated that the Notch ligand Delta-1 regulates membrane-bound IL6R expression in myeloid progenitor cells in vitro ${ }^{53}$. These studies prompted us to investigate the relationship between Il6r and Notch in HSC development. We performed loss-of-function experiments with Il6r and Il6 in transgenic tp1:eGFP embryos to track cells with active Notching signaling ${ }^{54}$. We did not observe a reduced number of tp1:eGFP ${ }^{+}$; $k d r l: m$ Cherry $^{+}$HSCs in Il6- and Il6r-deficient embryos in the aortic floor at 32-36 hpf (Fig. 4a, b). The results indicate that Il6r is not upstream of Notch in HSC specification.

If Il6r lies downstream of Notch signaling, then the HSC defect through inhibiting Notch signaling should be rescued by the ectopic expression of il6r. We first treated embryos at $12 \mathrm{hpf}$ using the Notch inhibitor DAPT to block Notch intracellular domain release from the plasma membrane, which led to the production of fewer HSCs in these embryos than in DMSO-treated siblings (Fig. 4c, d) ${ }^{14,55}$. In contrast, the enforced expression of il6r indeed rescued the depletion of $c m y b^{+}$HSCs along the DA region in DAPT-treated embryos at $48 \mathrm{hpf}$ (Fig. 4c, d). A similar result was observed in $c d 41$ :eGFP transgenic embryos at $72 \mathrm{hpf}$ (Fig. 4e, f), suggesting that Il6r may act downstream of Notch signaling in HSC development. 


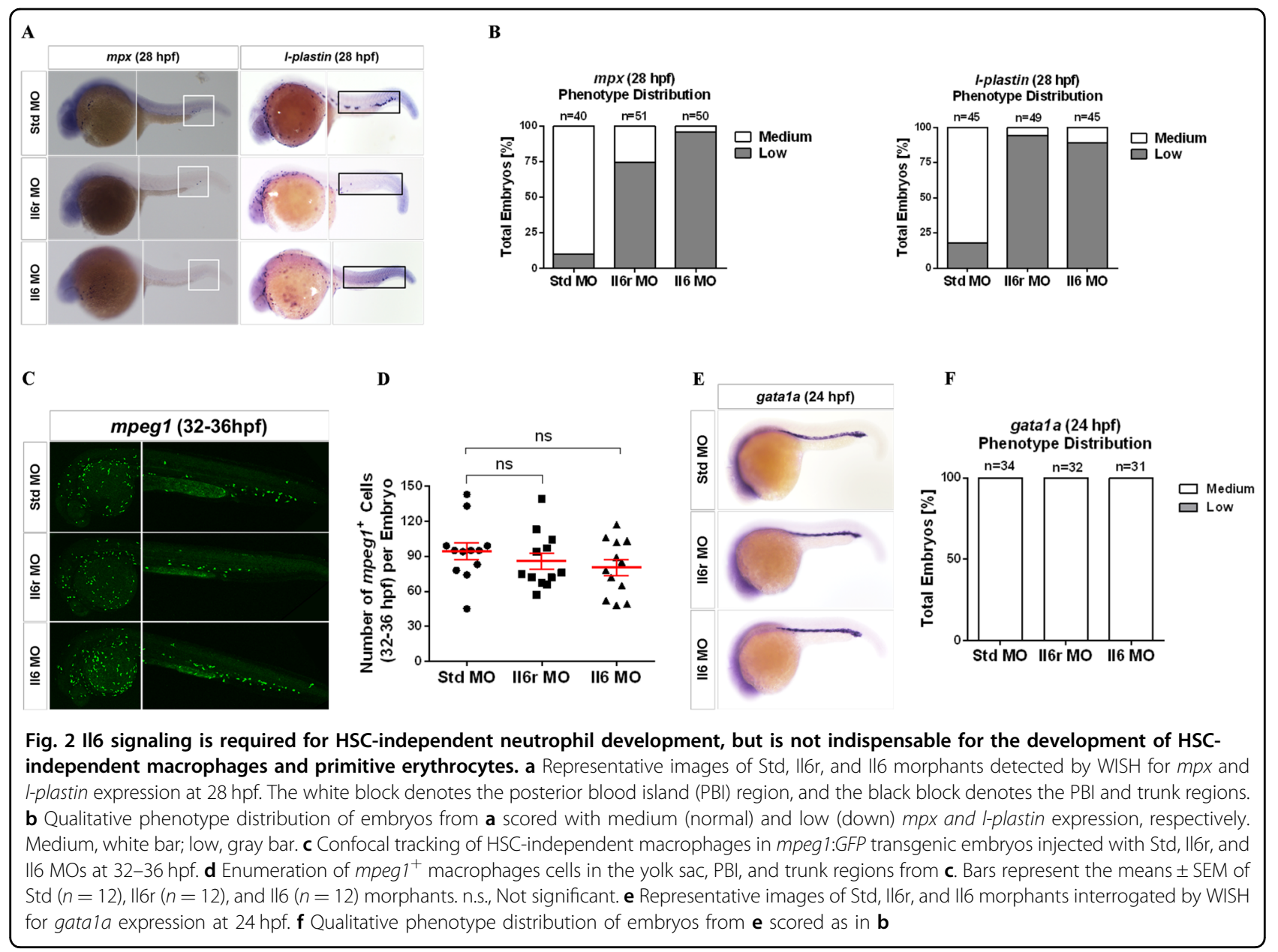

Notch1a promotes HSC specification in zebrafish embryos $^{13}$. Notch1a-dependent gata $2 b$ is required to initiate runx 1 expression in the $\mathrm{HE}^{56}$. Thus, we attempted to explore potential mechanisms by which Notch1a induced $i l 6 r$ activation. We isolated $f\left(i 1 a^{+}\right.$ECs by FACS from $28 \mathrm{hpf}$ transgenic flila:eGFP Notch1a-deficient embryos and performed qPCR for il6r, gata2b, and the known Notch target gene hey1. Intriguingly, all the transcripts were significantly lower in Notch1a morphants compared to controls (Fig. 4g). Additionally, Notch signaling is required downstream of Tnf $\alpha$ function during HSC emergence ${ }^{13}$. To investigate the functional relationship between Tnf $\alpha$ and Il6r, we enforced the expression of il6r in Tnfo-deficient embryos, and $\operatorname{runx} 1^{+}$cells in the aortic floor were restored at $28 \mathrm{hpf}$ (Fig. 4h, i). Together, these results indicate that Notch1a indeed functions upstream of il6r by regulating its expression within the HE to specify HSC fate.

\section{HSC-independent neutrophils are the key source of II6}

In adult organisms, IL6 is produced by almost all mature immune cells and HSPCs. ${ }^{11}$ While in the early development of zebrafish embryos, myeloid cells from primitive hematopoiesis and EMPs may be the key source of Il6 before HSC emergence. To investigate this hypothesis, we isolated mpx:GFP neutrophils and mpeg1: GFP MFs from embryos at $30 \mathrm{hpf}$ by FACS and performed qPCR to detect the expression of il6, il6r, gp130, and myeloid-specific factors, including pu.1, mpx, and csf1ra (Fig. 5a, b). Compared with the cells of whole embryos, both mpx- and mpeg1-positive cells had higher expression of $i l 6$ and il6r. This result suggests that HSC-independent myeloid cells produce a large amount of Il6.

To elucidate whether HSC-independent myeloid cells were the key source of Il6 needed for HSC emergence, we utilized a pu.1 MO to ablate myeloid cells ${ }^{38}$, and then used the panleukocyte marker l-plastin by WISH to validate the efficiency of the MO (Fig. S3). Following the ablation of myeloid cells, the expression of runx 1 in the DA region was significantly lower in pu.1 morphants compared to their control siblings (Fig. 5c, d). To further clarify which myeloid population was responsible for the reduction in HSC number, we utilized an irf8 $\mathrm{MO}^{13,37]}$, which drives myeloid progenitor development almost 


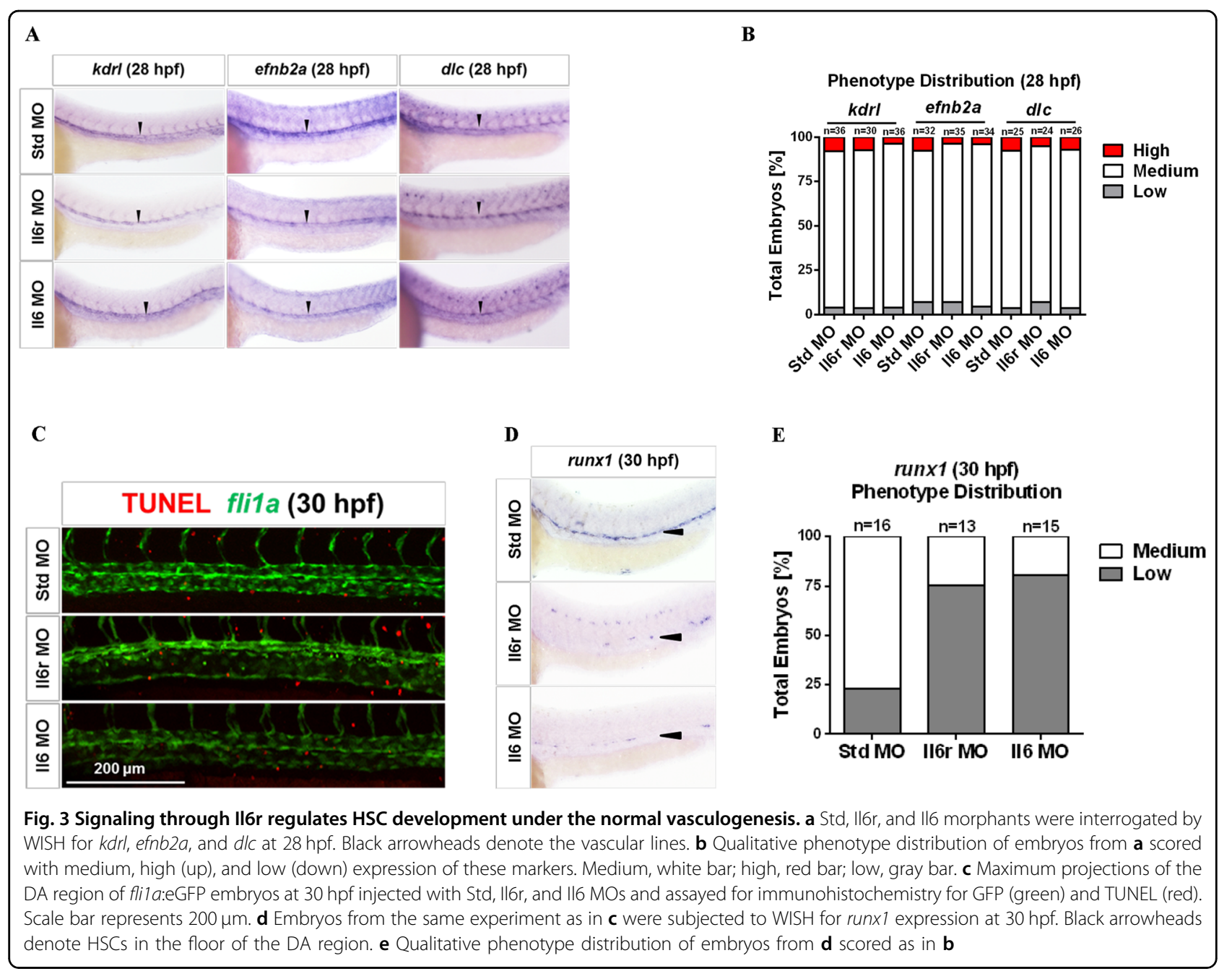

entirely into neutrophils. Irf8 MO efficiency was validated using the neutrophil marker $m p x$ at $29 \mathrm{hpf}$ (Fig. 5e, f). Following the elevated number of neutrophils in the PBI and trunk regions, runx 1 within the DA region was significantly higher in Irf8-deficient embryos at $29 \mathrm{hpf}$ compared to their control siblings. In agreement with our il6 expression data, this result suggests that neutrophils may be the key source of Il6 needed for HSC emergence. As such, we examined il6 expression in Pu.1- and Irf8 -deficient embryos. The expression of $i l 6$ was decreased in Pu.1 morphants and increased in Irf8 morphants (Fig. 5g). Furthermore, simultaneously inhibiting the expression of il6 and irf8 resulted in a lack of runx 1 expression, with a decreased number of neutrophils in the same regions (Fig. $5 \mathrm{e}, \mathrm{f})$. These results indicate that il6 derived from myeloid cells is necessary for HSC specification, and neutrophils are the key source of Il6. In addition, Il6 signaling is important for the development of HSC-independent neutrophils in the PBI and trunk regions.

\section{Discussion}

Inflammatory factors play an important role in adult HSPC proliferation and differentiation into mature immune cells under stress-induced hematopoiesis ${ }^{57,58}$. Indeed, short-term HSCs and MPPs can produce multiple cytokines at a single-cell resolution ${ }^{11}$. However, for a long time, it remains unknown whether proinflammatory signaling could regulate embryonic HSC development. Most recently, several research groups have demonstrated that proinflammatory factors, such as TNF- $\alpha$, IFN $-\gamma$, and TLR4, positively regulate HSC specification and emergence using zebrafish embryos ${ }^{12-14}$. As such, we also utilized the animal model to show that the depletion of Il6 and its cognate receptor Il6r leads to a failure of HSC emergence. These results support the hypothesis that HSCs evolved the capability of directly responding to proinflammatory factor signaling in embryonic development to defend against pathogenic infection after birth in almost all vertebrates. 


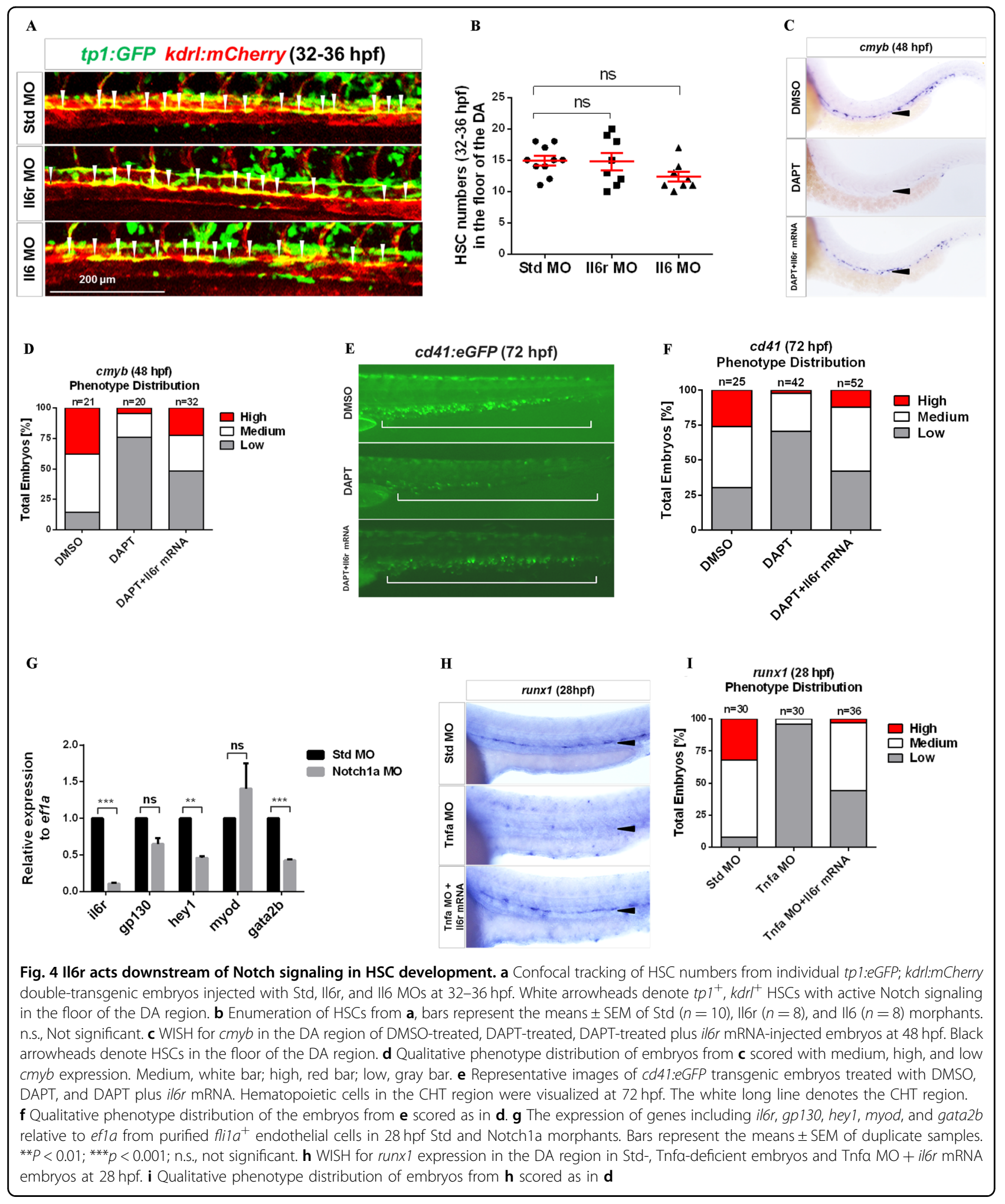

Our study demonstrates that blocking Il6 signaling has a significant impact on HSC production, while two previous studies showed that Il6- and Gp130-mutant adult mice had slight defects of HSPCs in terms of proliferation and differentiation ${ }^{23,24}$. Genetic compensation can partly explain these phenomena. MOs for knocking down targeted genes in lower-vertebrate animal models can effectively avoid compensatory 


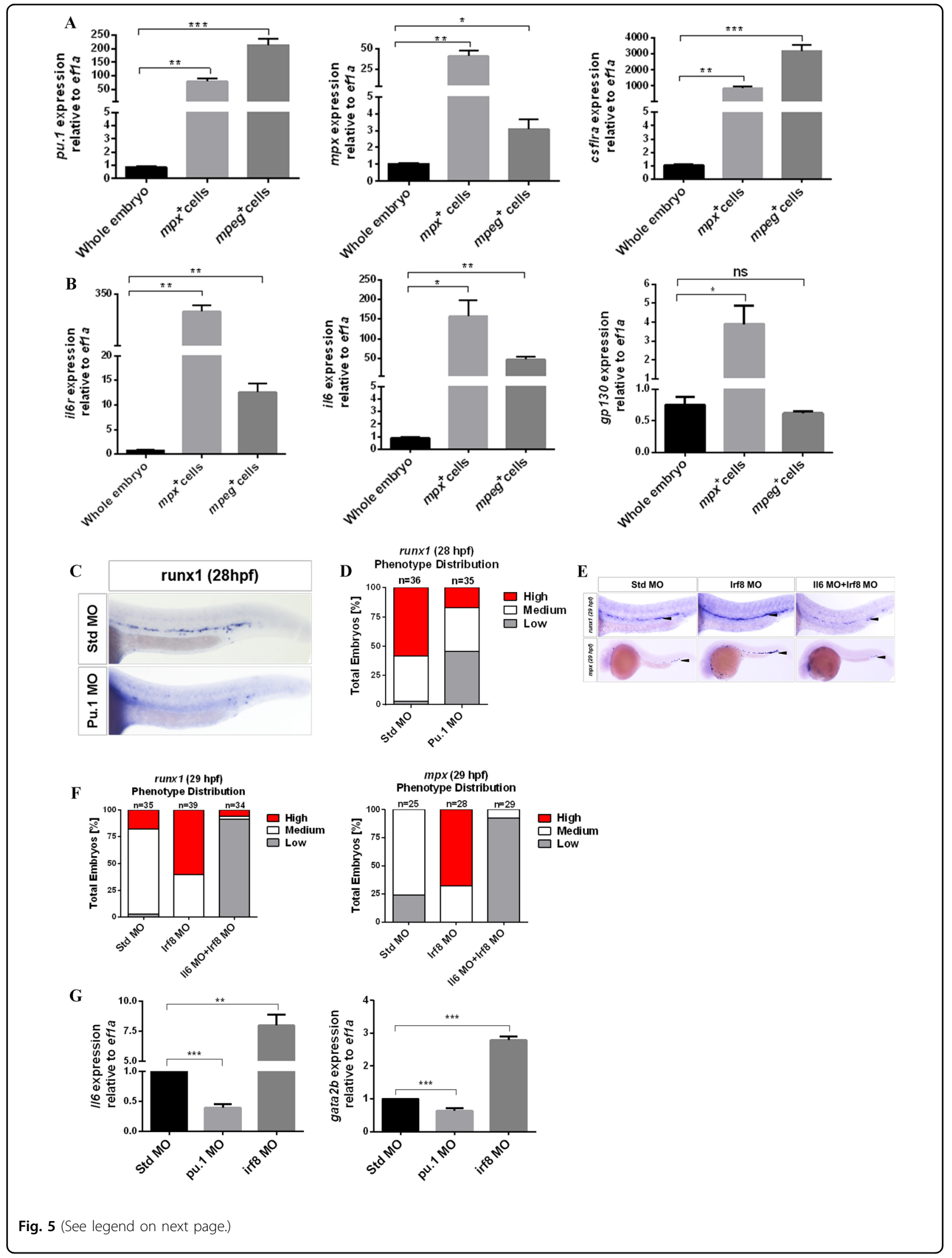


(see figure on previous page)

Fig. 5 HSC-independent neutrophils play a key role in HSC specification. a, b qPCR for detecting the expression of genes, including pu. 1, csf1ra, mpx, il6r, il6, and gp130, from purified HSC-independent macrophages (mpeg1:GFP ${ }^{+}$) and neutrophils $\left(m p x: G F P^{+}\right)$at 30 hpf. Expression was normalized to ef1 $a$ and is presented relative to whole-embryo expression. Bars represent the means \pm SEM of duplicate samples. ${ }^{*} P<0.05$; ${ }^{* *} p<0.01$; ${ }^{* * *} p<0.001$; n.S., not significant. c Std and Pu.1 morphants were examined by WISH for runx 1 expression at 28 hpf. d Qualitative phenotype distribution of embryos from c scored with medium, high, and low runx1 expression. Medium, white bar; high, red bar; low, gray bar. e Std, Irf8, and 116 + Irf8 morphants were examined by WISH for runx 1 and $m p x$ at 29 hpf. Black arrowheads denote runx $1^{+}$HSCs in the DA region and $m p x^{+}$ neutrophils in the PBI and trunk regions. $\mathbf{f}$ Qualitative phenotype distribution of embryos from e scored as in $\mathbf{d}$. $\mathbf{g}$ Whole-embryo expression of il6 and gata2b relative to ef1 $a$ in Std, Pu.1, and Irf8 morphants at $28 \mathrm{hpf}$. Bars represent the means \pm SEM of duplicate samples. ${ }^{* *} P<0.01 ;{ }^{* * *} p<0.001$

phenomena $^{59,60}$. Therefore, we utilized the zebrafish embryo model to comprehensively explore the function of Il6 signaling in HSC emergence.

In our study, we identified that the HSC program can be rescued in Notch1-inhibited embryos by the ectopic expression of $i l 6 r$, implying that $i l 6 r$ lies downstream of Notch in HSC specification. We demonstrated that Notch1a activates $i l 6 r$ by regulating its expression. In addition, we confirmed that Il6r acts downstream of Tnfo signaling. However, our study had some limitations. First, it remains to be determined whether Stat $3 / 1$ or other potential molecules might be downstream molecules of Il $6 \mathrm{r}^{14}$. Second, to our knowledge, Il6 signaling can be divided into classic signaling and trans-signaling pathways ${ }^{15}$. Classic IL6R receptor signaling refers to the activation of cells mediated via the membrane-bound IL6R subunit. Trans-signaling denotes a process in which a soluble IL6R/IL6 complex binds to cells expressing only the GP130 subunit. Further studies will be required to determine which Il6 signaling participates in the process of HSC production.

With regard to the source of II6, one study showed that murine AGM-derived stromal cells could secrete multiple cytokines, including $16^{61}$. Due to the lack of a recognized panel of specific protein markers, we failed to isolate these stromal cells. According to recent evidence, HSCs produce similar amounts of Il6 as vascular ECs or whole embryos ${ }^{28,41}$, so we deduced that HSC specification depends mainly on the paracrine effect of Il6. Indeed, we discovered for the first time that HSCindependent myeloid cells from primitive hematopoiesis and definitive EMPs are a major source of Il6. Neutrophils make a greater contribution to the source. Collectively, although sufficient evidence is available, it is necessary to precisely assess the contributions of different kinds of cells secreting Il6 using tissue-specific gene-knockout animal models.

In addition, we found an unexpected role for Il6 signaling in HSC-independent neutrophil development in the PBI and trunk regions. However, a similar function for Il6 signaling is not observed in primitive myeloid cells in the yolk sac. These results suggest that HSC-independent myeloid cells in different embryonic regions have different developmental orientations and functions in the adult immune system ${ }^{62,63}$. More studies are needed to elucidate the function of Il6 signaling in primitive and intermediate hematopoiesis.

In conclusion, our results suggest that Il6 signaling plays a pivotal role in HSC specification and emergence in zebrafish embryos. This information will be important for optimizing the strategy of HSC regeneration using the HE in vitro. However, it will still be a great challenge for the field to integrate various kinds of proinflammatory factor inputs in terms of the spatial and temporal requirements. More studies are necessary to optimize the protocol of HSC regeneration for HSC transplantation.

\section{Acknowledgements \\ This study was supported by the National Key Basic Research Program of China (No. 2015CB964900), Funds for International Cooperation and Exchange of the National Natural Science Foundation of China (No. 81520108002), the General Program of the National Natural Science Foundation of China (No. 81570098 and 8147158), and the Key Program of the National Natural Science Foundation of China (No. 81730008). We thank Zhipeng Ma and Xuye He for technical assistance and the anonymous reviewers for their fruitful comments We also thank the English editor for editing our manuscript appropriately.}

\section{Author details \\ 'Bone Marrow Transplantation Center, The First Affiliated Hospital, Zhejiang University School of Medicine, No. 79 Qingchun Road, 310003 Hangzhou, China. ${ }^{2}$ Institute of Hematology, Zhejiang University, No. 866 Yuhangtang Road, Hangzhou 310058, China. ${ }^{3}$ Zhejiang Engineering Laboratory for Stem Cell and Immunity Therapy, No. 866 Yuhangtang Road, Hangzhou 310058, China. ${ }^{4}$ Stem Cell Institute, Zhejiang University, No. 866 Yuhangtang Road, Hangzhou 310058, China. ${ }^{5}$ Department of Hematology, the Second Clinical Medical College, Shanxi Medical University, No. 382 Wuyi Road, 030000 Taiyuan, China}

\section{Authors' contributions}

H.H., R.X.T., H.H.L. and S.Y.C. conceived and designed the study. R.X.T. constructed the plasmids and prepared the probes. R.X.T., H.H.L. and S.Y.C. performed the WISH and other experiments. Z.Y.L., W.S., B.S.W., Y.M.T. and W.Y Z. contributed to the acquisition of data. H.H., R.X.T., H.H.L. and S.Y.C. analyzed and interpreted the data. R.X.T., H.H.L. and S.Y.C. wrote, reviewed, and revised the paper. H.H. and R.X.T. supervised the study.

\section{Data availability}

All data generated or analyzed during this study are included in this published article and the supplementary information.

Conflict of interest

The authors declare that they have no conflict ofinterest. 


\section{Publisher's note}

Springer Nature remains neutral with regard to jurisdictional claims in published maps and institutional affiliations.

Supplementary information accompanies this paper at https://doi.org/ 10.1038/s12276-019-0320-5.

Received: 4 August 2018 Revised: 19 April 2019 Accepted: 30 May 2019. Published online: 24 October 2019

\section{References}

1. Bertrand, J. Y. et al. Haematopoietic stem cells derive directly from aortic endothelium during development. Nature 464, 108-111 (2010).

2. Boisset, J. C. et al. In vivo imaging of haematopoietic cells emerging from the mouse aortic endothelium. Nature 464, 116-120 (2010).

3. de Bruijn, M. F., Speck, N. A., Peeters, M. C. \& Dzierzak, E. Definitive hematopoietic stem cells first develop within the major arterial regions of the mouse embryo. EMBO J. 19, 2465-2474 (2000).

4. Kissa, K. \& Herbomel, P. Blood stem cells emerge from aortic endothelium by a novel type of cell transition. Nature 464, 112-115 (2010).

5. Daniel, M. G., Pereira, C. F., Lemischka, I. R. \& Moore, K. A. Making a hematopoietic stem cell. Trends Cell Biol. 26, 202-214 (2016).

6. Ivanovs, A. et al. Human haematopoietic stem cell development: from the embryo to the dish. Development 144, 2323-2337 (2017).

7. Dzierzak, E. \& Bigas, A. Blood development: hematopoietic stem cell dependence and independence. Cell Stem Cell 22, 639-651 (2018).

8. $\mathrm{Xu}, \mathrm{Y}$. et al. A synthetic three-dimensional niche system facilitates generation of functional hematopoietic cells from human-induced pluripotent stem cells. J. Hematol. Oncol. 9, 102 (2016).

9. King, K. Y. \& Goodell, M. A. Inflammatory modulation of HSCs: viewing the HSC as a foundation for the immune response. Nat. Rev. Immunol. 11, 685 (2011).

10. Nagai, Y. et al. Toll-like receptors on hematopoietic progenitor cells stimulate innate immune system replenishment. Immunity 24, 801-812 (2006).

11. Zhao, J. L. et al. Conversion of danger signals into cytokine signals by hematopoietic stem and progenitor cells for regulation of stress-induced hematopoiesis. Cell Stem Cell 14, 445-459 (2014).

12. He, Q. et al. Inflammatory signaling regulates hematopoietic stem and progenitor cell emergence in vertebrates. Blood 125, 1098-1106 (2015).

13. Espín-Palazón, R. et al. Proinflammatory signaling regulates hematopoietic stem cell emergence. Cell 159, 1070-1085 (2014).

14. Sawamiphak, S., Kontarakis, Z. \& Stainier, D. R. Interferon gamma signaling positively regulates hematopoietic stem cell Emergence. Dev. Cell 31, 640-653 (2014).

15. Stachura, D. L. et al. The zebrafish granulocyte colony-stimulating factors (Gcsfs): 2 paralogous cytokines and their roles in hematopoietic development and maintenance. Blood 122, 3918-3928 (2013).

16. Li, Y. et al. Inflammatory signaling regulates embryonic hematopoietic stem and progenitor cell production. Genes Dev. 28, 2597-2612 (2014).

17. Hunter, C. A. \& Jones, S. A. IL-6 as a keystone cytokine in health and disease. Nat. Immunol. 16, 448-457 (2015).

18. Murakami, M. 1, Kamimura, D. \& Hirano, T. Pleiotropy and specificity: insights from the interleukin 6 family of cytokines. Immunity 50, 812-831 (2019).

19. Hibi, M. et al. Molecular cloning and expression of an IL-6 signal transducer, gp130. Cell 63, 1149-1157 (1990).

20. Taga, T., Kawanishi, Y., Hardy, R. R., Hirano, T. \& Kishimoto, T. Quantitation, specificity, distribution, and regulation of their expression. J. Exp. Med. 166, 967-981 (1987)

21. Taga, $T$. et al. Interleukin-6 triggers the association of its receptor with a possible signal transducer, gp130. Cell 58, 573-581 (1989).

22. Yamasaki, K. et al. Cloning and expression of human interleukin 6 (BSF-2/ IFNB2) receptor. Science 241, 825-828 (1988).

23. Yoshida, K. et al. Targeted disruption ofgp130, a common signal transducer for the interleukin 6 family of cytokines, leads to myocardial and hematological disorders. Proc. Natl. Acad. Sci. USA 93, 407-411 (1996).

24. Bernad, A. et al. Interleukin-6 is required in vivo for the regulation of stem cells and committed progenitors of the hematopoietic system. Immunity 1, 725-731 (1994).

25. Stachura, D. L. et al. Zebrafish kidney stromal cell lines support multilineage hematopoiesis. Blood 114, 279-289 (2009).
26. Doulatov, S. et al. Induction of multipotential hematopoietic progenitors from human pluripotent stem cells via respecification of lineage-restricted precursors. Cell Stem Cell 13, 459-470 (2013).

27. Kennedy, M. et al. T lymphocyte potential marks the emergence of definitive hematopoietic progenitors in human pluripotent stem cell differentiation cultures. Cell Rep. 2, 1722-1735 (2012).

28. Lim, S. E. et al. HIF1a-induced PDGFRß signaling promotes developmental HSC production via IL-6 activation. Exp. Hematol. 46, 83-95 (2017).

29. McGrath, K. E. et al. Distinct sources of hematopoietic progenitors emerge before HSCs and provide functional blood cells in the mammalian embryo. Cell Rep. 11, 1892-1904 (2015).

30. Frame, J. M., McGrath, K. E. \& Palis, J. Erythro-myeloid progenitors: "definitive" hematopoiesis in the conceptus prior to the emergence of hematopoietic stem cells. Blood Cells Mol. Dis. 51, 220-225 (2013).

31. Hoeffel, G. et al. C-Myb(+) erythro-myeloid progenitor-derived fetal monocytes give rise to adult tissue-resident macrophages. Immunity 42, 665-678 (2015).

32. Davidson, A. J. \& Zon, L. I. The 'definitive' (and 'primitive') guide to zebrafish hematopoiesis. Oncogene 23, 7233-7246 (2004).

33. Clements, W. K. \& Traver, D. Signalling pathways that control vertebrate haematopoietic stem cell specification. Nat. Rev. Immunol. 13, 336-348 (2013).

34. Rasighaemi, P., Basheer, F., Liongue, C. \& Ward, A. C. Zebrafish as a model for leukemia and other hematopoietic disorders. J. Hematol. Oncol. 8, 29 (2015).

35. Liu, X. et al. DNA methyltransferase 1 functions through C/ebpa to maintain hematopoietic stem and progenitor cells in zebrafish. J. Hematol. Oncol. 8, 15 (2015).

36. Westerfield, M. A guide for the laboratory use of zebrafish (Danio rerio). The Zebrafish Book. 4 (edn.), (The University of Oregon Press: Eugene, 2000).

37. Li, L., Jin, H., Xu, J., Shi, Y. \& Wen, Z. Irf8 regulates macrophage versus neutrophil fate during zebrafish primitive myelopoiesis. Blood 117, 1359-1369 (2011).

38. Rhodes, J. et al. Interplay of pu.1 and gata1 determines myelo-erythroid progenitor cell fate in zebrafish. Dev. Cell 8, 97-108 (2005).

39. Thisse, C. \& Thisse, B. High-resolution in situ hybridization to whole-mount zebrafish embryos. Nat. Protoc. 3, 59-69 (2008).

40. Wang, L. et al. Fev regulates hematopoietic stem cell development via ERK signaling. Blood 122, 367-375 (2013).

41. Zhou, F. et al. Tracing haematopoietic stem cell formation at single-cell resolution. Nature 533, 487-492 (2016).

42. Burns, C. E., Traver, D., Mayhall, E., Shepard, J. L. \& Zon, L. I. Hematopoietic stem cell fate is established by the Notch-Runx pathway. Genes Dev. 19, 2331-2342 (2005).

43. Bertrand, J. Y., Kim, A. D., Teng, S. \& Traver, D. CD41+ c-myb+ precursors colonize the zebrafish pronephros by a novel migration route to initiate adult hematopoiesis. Development 135, 1853-1862 (2008).

44. Chen, M. J., Yokomizo, T., Zeigler, B. M., Dzierzak, E. \& Speck, N. A. Runx1 is required for the endothelial to haematopoietic cell transition but not thereafter. Nature 457, 887-891 (2009).

45. Langenau, D. M. et al. In vivo tracking of T cell development, ablation, and engraftment in transgenic zebrafish. Proc. Natl. Acad. Sci. USA 101, 7369-7374 (2004).

46. Gering, M. \& Patient, R. Hedgehog signaling is required for adult blood stem cell formation in zebrafish embryos. Dev. Cell 8, 389-400 (2005).

47. Bertrand, J. Y. et al. Definitive hematopoiesis initiates through a committed erythromyeloid progenitor in the zebrafish embryo. Development 134, 4147-4156 (2007)

48. Lawson, N. D. et al. Notch signaling is required for arterial-venous differentiation during embryonic vascular development. Development $\mathbf{1 2 8}$ 3675-3683 (2001)

49. Kobayashi, I. et al. Jam1a-Jam2a interactions regulate haematopoietic stem cell fate through Notch signalling. Nature 512, 319-323 (2014).

50. Kumano, K. et al. Notch1 but not Notch2 is essential for generating hematopoietic stem cells from endothelial cells. Immunity 18, 699-711 (2003).

51. Robert-Moreno, A., Espinosa, L., de la Pompa, J. L. \& Bigas, A. RBPjkappadependent Notch function regulates Gata2 and is essential for the formation of intra-embryonic hematopoietic cells. Development 132, 1117-1126 (2005).

52. Robert-Moreno, A. et al. Impaired embryonic haematopoiesis yet normal arterial development in the absence of the Notch ligand Jagged1. EMBO J. 27, 1886-1895 (2008).

53. Csaszar, E. et al. Blood stem cell fate regulation by Delta-1-mediated rewiring of IL-6 paracrine signaling. Blood 123, 650-658 (2014). 
54. Parsons, M. J. et al. Notch-responsive cells initiate the secondary transition in larval zebrafish pancreas. Mech. Dev. 126, 898-912 (2009).

55. Kim, H. S., Jeong, H., Lim, S. O. \& Jung, G. Snail inhibits Notch1 intracellular domain mediated transcriptional activation via competing with MAML1. Biochem. Biophys. Res. Commun. 433, 6-10 (2013).

56. Butko, E. et al. Gata2b is a restricted early regulator of hemogenic endothelium in the zebrafish embryo. Development 142, 1050-1061 (2015).

57. Takizawa, H., Boettcher, S. \& Manz, M. G. Demand-adapted regulation of early hematopoiesis in infection and inflammation. Blood 119, 2991-3002 (2012).

58. Baldridge, M. T., King, K. Y. \& Goodell, M. A. Inflammatory signals regulate hematopoietic stem cells. Trends Immunol. 32, 57-65 (2011).

59. Espin-Palazon, R., Weijts, B., Mulero, V. \& Traver, D. Proinflammatory signals as fuel for the fire of hematopoietic stem cell emergence. Trends Cell Biol. $\mathbf{2 8}$ 58-66 (2018).
60. Ma, Z. et al. PTC-bearing mRNA elicits a genetic compensation response via Upf3a and COMPASS components. Nature 568, 259-263 (2019).

61. Xu, M. J. et al. Stimulation of mouse and human primitive hematopoiesis by murine embryonic aorta-gonad-mesonephros-derived stromal cell lines. Blood 92, 2032-2040 (1998).

62. Gomez Perdiguero, E. et al. Tissue-resident macrophages originate from yolksac-derived erythro-myeloid progenitors. Nature 518, 547-551 (2015).

63. $\mathrm{Xu}, \mathrm{J}$. et al. Temporal-spatial resolution fate mapping reveals distinct origins for embryonic and adult microglia in zebrafish. Dev. Cell 34, 632-641 (2015). 